\title{
An Analysis of Emotional Tendency Under the Network Public Opinion: Deep Learning
}

\author{
Jinze Li, Yizhen Wang and Jun Wang \\ China People's Police University, School of Intelligence Policing, Langfang, Hebei 065000, China \\ E-mail: j9z571@163.com
}

Keywords: network public opinion, emotional orientation, deep learning, OCC model, convolutional neural network

Received: January 5, 2021

\begin{abstract}
Network public opinion refers to the common opinion with tendency and influence formed by the public on certain social events through the Internet. Due to the complexity of interest relations, network public opinion is likely to cause difficulties for individuals, enterprises, or governments. To control the public's emotional tendency to social events, this study designed an OCC sentiment rule system to label the network public opinion case base. The text representation method is Word2Vec in deep learning, and the convolution neural network is used to construct the sentiment tendency analysis model under the network public opinion. Taking the case of Dolce \& Gabbana humiliation incident, Xiangshui explosion incident, and baixiangguo girl's murder as the research cases, the accuracy of the model in identifying the above three events was $85.87 \%, 73.65 \%$, and $85.87 \%$, respectively, under the optimal parameters setting. The experimental results show that the proposed method can improve the accuracy of emotion recognition by $3.00 \%$ 8.00\% compared with the manual annotation method, i.e., the network public opinion sentiment orientation recognition model constructed in this study has a high recognition accuracy and can be used to assist relevant departments in detecting network public opinion.
\end{abstract}

Povzetek: Z globokim učenjem je narejena analiza mnenja uporabnikov omrežja o določeni tematiki.

\section{Introduction}

The dissemination and governance of network public opinion is an important part of government work, and the analysis of network public opinion sentiment tendency is the basic work to eliminate the network environment. At present, scholars have made a detailed analysis on the generation mechanism, evolution mechanism, impact on social economy, and guidance mechanism of network public opinion. Wu et al. used the social network analysis (SNA) framework to analyze the generation mechanism and evolution process of network public opinion [1]. Huang analyzed the changing trend of public policy under the sudden network public opinion event [2]. Song et al. analyzed the social transformation crisis and economic risk generated by network public opinion events [3]. Zhang's team proposed a network public opinion monitoring, tracking, analysis, and guidance system based on extensive investigation of existing network public opinion monitoring research [4]. The academic community has realized the emotional analysis and prediction of network public opinion on a certain field of social events. Li et al. have realized real-time tracking, monitoring and comprehensive evaluation of public opinion information on agricultural product quality and safety in the form of intelligent platform assisted by manual [5]. Based on the authenticity of network information dissemination, Hong's team realized the simulation of the propagation process of food safety network public opinion events [6]. At present, the research on the evolution mechanism of Internet public opinion and the analysis of emotional tendency about social events such as food, environment, and international disputes have been relatively perfect, but there are few studies on the universal model of emotional orientation analysis of network public opinion. Given the above situation, this study relies on the standard emotional rule system to construct the identification model of netizens' emotional orientation to realize a comprehensive and efficient understanding of the emotion contained in the information text.

\section{Construction of network public opinion sentiment tendency analysis model}

\subsection{Design of emotional rules of network public opinion}

In this study, the OCC model is used to construct emotion recognition rules. The final output of this model is the emotional tendency of netizens to a certain social event or social phenomenon. The generation process can be divided into four parts: classification, quantification, mapping, and expression [7, 8]. The evaluation criteria of the OCC model are event result, object behavior, and object image. The information receiver will make an emotional judgment on these three components. If the evaluation subject pays more attention to the event results, 
the OCC model needs to focus on analyzing the evaluation subject's evaluation of the target; if the evaluation subject pays more attention to the behavior of the object, the model focuses on the behavior criterion of the evaluation subject; if the evaluation subject pays more attention to the image of the object, the model pays more attention to the emotional attitude of the evaluation subject to the evaluation object. The satisfaction degree of the event result, the behavior standardization of the object, and the attitude towards the target object may affect the emotional color of the information receiver. The standard design of emotional orientation recognition rules should not only include common emotional orientation words but also consider the language context of the comment. In this study, the judgment of the information receiver's emotional orientation is mainly based on the semantics of the information receiver's comment events and the logical relationship between sentences and the emotional orientation words.

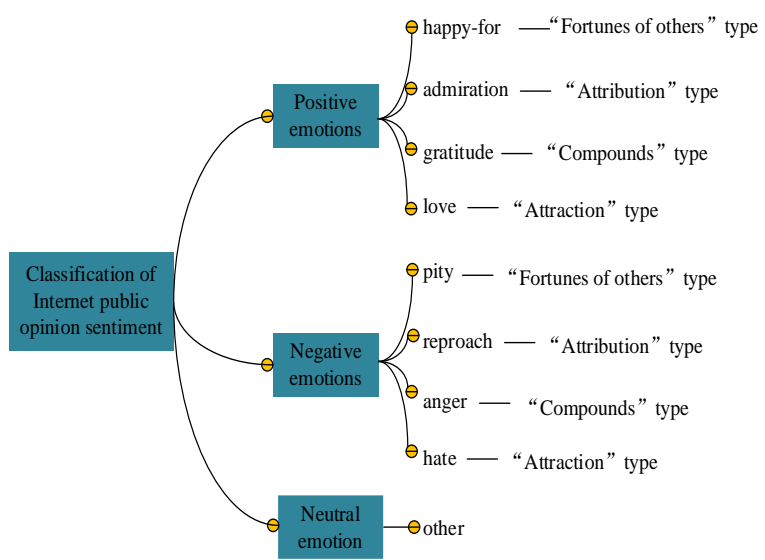

Figure 1: Classification and mapping of the emotional tendency of Internet public opinion.

The emotional orientation recognition model constructed in this study tends to provide a reference for relevant departments to monitor the network environment and control the direction of public opinion; thus, it is more inclined to understand the negative comments of netizens on social events or social phenomena. After understanding the emotional tendency of netizens to the event, the relevant departments can conduct targeted public opinion guidance to prevent large-scale network violence and affect social harmony [9]. Considering the closed-loop principle and the requirements of fine emotional granularity, this study creatively divides the emotional orientation of evaluation subjects into three categories: positive, medium, and negative. Referring to relevant literature, after screening and identifying, eight basic emotions are identified as the basic emotional types of online public opinion, as shown in Figure 1. These eight kinds of emotions are happiness, appreciation, regret, condemnation, anger, hate, love, and thanks for others. Appreciation, happiness for others, love and gratitude are mapped into positive emotions; anger, regret, condemnation, and hate are mapped to negative emotions; the comments that cannot identify emotional tendencies are mapped to neutral emotions [10].
When using the OCC model to identify the emotional orientation of online public opinion, it is necessary to analyze the correspondence between the subjective comment extractor evaluation standard and the emotional rules according to the evaluation criteria published by the evaluation subject. According to the information shown in Figure 1, eight kinds of emotion rules are constructed in this study. These emotional rules can not be used as the input content of the subsequent recognition model directly, and they need to be transformed into functional forms that are easy to implement in text. This paper constructs a 9-dimensional emotional space and assigns emotional variables to each comment text. The calculation formula is:

Emotions $=\left[e_{\text {happy-for }}, e_{\text {pity }}, e_{\text {admiretion }}, e_{\text {reproach }}, e_{\text {gratitude }}, e_{\text {anger }}, e_{\text {love }}, e_{\text {other }}\right],(1)$

where Emotions in formula (1) represents the emotional variable of the text and ${ }^{e[0,1]}$ represents the value of each dimension of emotion.

Emtion $($ positive $)=$ Emtion $($ happy - for $) \cup$ Emtion(admiration $)$ $\cup$ Emtion (gratitude) $\cup$ Emtion (love)

Emtiom $($ negative $)=$ Emtion $($ pity $) \cup$ Emtion $($ reporach $)$ $\cup$ Emtion(anger) $\cup$ Emtion (hate)

Emtion (neutral) $=1-$ Emtion ( positive $)-$ Emtiom (negative $)$

Equation (2) is the positive, middle, and negative emotional rules constructed in this study.

The emotion of "fortunes for other" is event-driven, and the emotion of the information receiver is based on the result of an event; thus, satisfaction is an important variable of emotion evaluation.

Emtion $($ happy - for $)=$ EventConsequece $\left(\right.$ txt $\left._{i}, e\right) \cap$ FocusOn $(e, s)$ $\cap\left(\right.$ DesireOf $(o, e) \cup$ DesireOf $_{s}$ DeservedOf $\left._{o} e\right)(3)$

Emtion $($ pity $)=$ EventConsequece $\left(\right.$ txt $\left._{i}, e\right) \cap$ FocusOn $(e, s)$

$$
\cap\left(\text { DesireOf }\left(o_{i}, \sim e\right) \cup \text { DesireOf }_{s} \sim \text { DeservedOf }_{o} e\right)
$$

Equation (3) is the recognition rule of "happy for others". When the evaluator $S$ thinks that the result of event $e$ meets the objective expectation of evaluation object $O$, the emotion of the text can be recognized as "happy for others". Equation (4) is the recognition rule of regret emotion. When the evaluator $S$ thinks that the result of event $e$ does not meet the objective expectation of evaluation object $O$, the emotion of the text can be recognized as "regret".

"Attribution" emotion is a multi-type emotion. When the behavior of the evaluation object conforms to the behavior standard of the evaluation subject, the evaluation subject will produce positive emotions such as appreciation to the evaluation object. In this study, the IdealOf function is used to judge the emotional orientation of object behavior.

Emtion $($ admiration $)=$ EventAction $\left(\right.$ txt $\left._{i}, a\right) \cap$ FocusOf $(a, o)$ $\cap$ MotivationOf $(a, m) \cap$ IdealOf $(s, m$, positive $)(5)$

Emtion $($ reproach $)=$ EventAction $\left(\right.$ txt $\left._{i}, a\right) \cap \operatorname{ActionOf}(a, o)$ $\cap$ MotivationOf $(a, m) \cap$ IdealOf $(s, m$, negative $)(6)$

Equations (5) and (6) are the recognition rules of "appreciation" and "regret", respectively. If IdeaOf is positive, the emotion of the text is "appreciation"; if IdeaOf is negative, the result of text recognition is "regret".

"Compounds" emotion means compound emotion. When the event result and object behavior interact, the 
information receiver may have multiple emotions for the information. When the evaluator associates his own experience, or the event touches his own interests through the event, the information receiver may have the emotion of "gratitude" or "anger", and the recognition rules are:

Emtion $($ gratitude $)=$ Event $\left(\right.$ txt $\left.{ }_{i}, e, a\right) \cap A c t i o n O f(a, o) \cap$ MotivationOf $(a, m) \cap$ IdealOf $(s, m$, positive $) \cap \operatorname{DesireOf}(s, e)$

Emtion $($ anger $)=\operatorname{Event}\left(t x t_{i}, e, a\right) \cap \operatorname{ActionO} f(a, o) \cap \operatorname{MotivationOf}(a, m) \cap$

$$
\text { IdealOf }(s, m, \text { negative }) \cap \text { DesireOf }(s, \sim e)
$$

The source of "attraction" emotion is the preference and dislike of the information receiver to the object image. The information receiver will ignore the event results and the object behavior and express their own opinions on the object image. This kind of emotion is very common in netizens' comments on entertainment events. This type of emotion is mainly "like" and "hate". The recognition rules are:

Emtion $($ love $)=$ EventObject $\left(\right.$ txt $\left._{i}, o\right) \cap$ AttractionOf $(s, o$, positive $),(9)$ Emtion $($ hate $)=$ EventObject $\left(\right.$ txt $\left._{i}, o\right) \cap$ AttractionOf $(s, o$, negative $)$. (10)

The emotional rules of network public opinion based on the OCC model constructed in this study are as follows. When the evaluation criterion of emotion classification is event result and the evaluation subject is satisfied with the results of others, there will be feelings of "happy for others"; when the evaluation subject is not satisfied with the results of others, there will be "regret" emotion; when the evaluation subject is not satisfied with its results, there will be "sad" emotion. When the evaluation subject is satisfied with its results, there will be "happy" emotion. When the evaluation object is taken as the evaluation standard, whether the evaluation subject likes the evaluation object or not determines the emotion as "like" or "hate". When the object's behavior is taken as the evaluation standard, the approval of the evaluation subject determines the emotion as "appreciation" or "condemnation". When the object's behavior and event consequences are taken as the comprehensive evaluation criteria and they are satisfied with their results and agree with the object's behavior, they will have "gratitude" emotion; when they are not satisfied with their results and do not agree with the object's behavior, they will have "anger" emotion.

\subsection{Identification of emotional tendency of Internet public opinion}

The popularity of the Internet enables the public to express their opinions freely on the public platform. At present, the market is full of a large number of social news software, which provides a lot of channels for the public to express opinions. Generally speaking, the online public opinion text for a social event or social phenomenon is more than 10000 or 100000 , covering multiple applications. The traditional classification method of Internet public opinion sentiment is manual annotation. Professionals summarize and analyze the comments made by Internet users with different software to judge the public's emotional tendency towards the event $[11,12]$. However, the recognition efficiency of this method is low, the recognition results are easily affected by the subjective emotions of the staff, and the accuracy of the recognition results is difficult to guarantee. In this study, the Word2Vec text representation method and convolutional neural network are used to construct the network public opinion sentiment rule recognition model; with the help of computers, the objective and rapid identification of network public opinion emotion can be realized [13, 14].

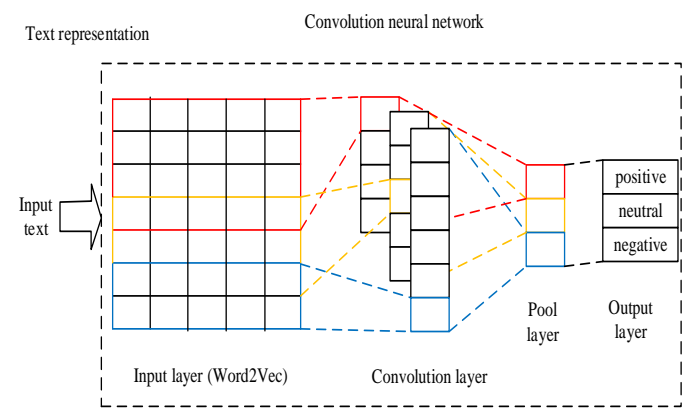

Figure 2: Recognition model of Internet public opinion sentiment rules.

The basic model of network public opinion sentiment rule recognition is shown in Figure 2. The core idea of the model is to predict the sentiment tendency of the text by calculating the vector matrix formed by the text. The key to extracting high-level abstract features hidden in text data using a convolutional neural network is to select the appropriate text representation method [15]. This study uses Word2Vec as the training model of word vector feature extraction. The model runs fast and can train hundreds of billions of words in 24 hours.
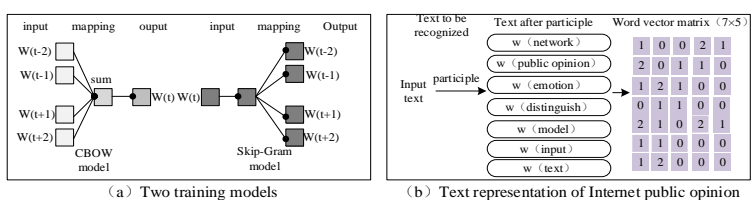

Figure 3: Representation method and training model of Internet public opinion text.

Figure 3 (a) is the logical block diagram of the Word2Vec training model. The training and prediction of the Word2Vec model is based on the CBOW (Continuous Bag of Words) model and skip-gram model $[16,17] .{ }^{w(t)}$ in the figure indicates the position ${ }^{t}$ of the current word in the text statement, and other words except the word form the context. The principle of word recognition in the Word2Vec model is as follows: the Word2Vec model uses word frequency as a leaf node, uses words with similar word frequency in the hidden layer to activate similar content, and word frequency is inversely proportional to the number of active hidden layers. Figure 3 (b) is an example of the output result of emotion recognition for a text. Assuming that the text has seven words and the word vector dimension generated by the Word $2 \mathrm{Vec}$ training model is 5 , the text represents a two-dimensional matrix of word vector with the result of $7 \times 5$. The matrix can be used as the input vector of the next classifier.

After the text is transformed into a vector matrix by the Word2Vec model, to predict the emotion of the text with the vector matrix, this study uses the convolutional neural networks $(\mathrm{CNN})$ to classify the emotional tendency 
of the subjective evaluation of the evaluation subject. The basic structure of convolution neural network includes convolution layer, pooling layer, full connection layer, and output layer $[18,19]$. The unique structure of a convolutional neural network has the characteristics of local connection, weight sharing, and time-space subsampling, which can effectively reduce the number of calculated weights and is suitable for solving the classification problem of multivariable. The operation process and output results of text data in the convolution layer and pooling layer of a convolution neural network are shown in Figure 4.

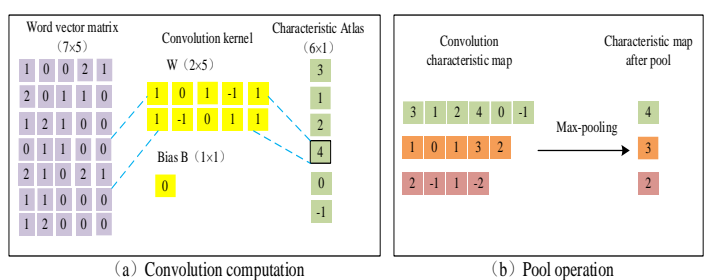

Figure 4: Operation of network public opinion information text in a convolution neural network.

Figure 4 (a) shows the operation of the convolution layer on information text. The convolution layer is a $2 \times 5$ convolution kernel, and the weight parameter matrix is generated randomly. The input text is a $7 \times 5$ feature matrix; after convolution processing, a $6 \times 1$ size feature map can be obtained.

The two-dimensional matrix generated by the Word2Vec model is the input of the convolution layer, the dimension of $7 \times 5$ vector matrix is reduced to $6 \times 1$ matrix, and the weight parameters are reduced, which reduces the operation difficulty and operation time of the convolution neural network. The two-dimensional matrix generated by the Word $2 \mathrm{Vec}$ model is the input of the convolution layer, the dimension of the $7 \times 5$ vector matrix is reduced to the $6 \times 1$ matrix. This operation reduces the weight parameters, reduces the difficulty of the convolution neural network operation, and greatly reduces the operation time. In the actual training of network public opinion information text, sometimes the value of the input word vector matrix does not change obviously, and the linear model lacks enough expression ability, which leads to the output deviation from the correct value. In this study, the nonlinear factors are introduced to distinguish the nonlinear data, and the relu function of the unsaturated nonlinear method is used as the activation function, so that the convolution neural network can effectively process the data $[20,21]$.

Figure 4 (b) is a schematic diagram of the operation of the pooling layer on information text. The pooling layer can maintain the invariance of the map to a certain extent after operations such as translation and scaling while retaining the text information, reducing the feature dimension, and improving the operation speed [22]. After pooling the $6 \times 1$ matrix output from the convolution layer, the number of neurons in the feature map remains unchanged, and the feature map of 7 words has six row elements. The pool forming process of pool layer pair characteristic map is:

$$
Z_{i}=f\left(W \square \operatorname{pool}\left(C_{i}\right)+B\right)
$$

where $\operatorname{pool}(\square)$ is the characteristic map after pooling treatment. In this study, the maximum value of neurons in each area is selected, and the maximum value is defined as $\operatorname{pool}_{\max }\left(C_{i}\right)=\max \left\{R_{x}\right\}$.

As shown in Figure 4 (b), the maximum characteristic elements of $6 \times 1,5 \times 1$, and $4 \times 1$ feature maps are 4,3 , and 2 , respectively. The expression of the full connection function of the convolution neural network is:

$$
Z=f\left(Z_{1}, Z_{2}, . . Z_{i}\right)
$$

After the full connection operation of the feature map is completed, the classification function is used to convert the output of the defined linear function into the sentiment category of network public opinion, and the proportion of positive, neutral, and negative emotional tendency of the text can be speculated. As this study focuses on the negative emotions of online public opinion texts, if the proportion of negative texts exceeds the sum of positive and neutral texts, the event will be regarded as having a greater negative impact on the society; if the proportion of positive texts exceeds the sum of negative texts and neutral texts, the event will have a positive and healthy impact on the society. The logic of updating parameters of the recognition module is as follows: some hidden nodes of the model do not participate in the recognition process, but their weight values will be retained and only participate in the recognition operation at the next sample input [23].

\section{An empirical analysis of the emotional tendency analysis model of Internet public opinion}

\subsection{Experimental design}

This study took Dolce \& Gabbana humiliation incident in November 2018, Xiangshui explosion in March 2019, and baixiangguo girl's murder in November 2020 as cases. The three incident numbers were DG_2018, XS_2019, and BXG_2020. This study grabbed 24808 text data for the 2018 event, 8856 data texts for the XS_2019 event, and 65537 data texts for the BXG_2020 event.

First of all, the obtained data were cleaned to remove the unclear and confusing texts. The effective data of the three events were 23043, 4573, and 16773, respectively. After cleaning, the effective data were labeled by sentiment classification to form the case database data set of network public opinion events. Data preprocessing mainly included word segmentation and data length processing of Chinese texts. The sample size ratio of training set and data set was 9:1. There were 20738, 4116, and 15096 data texts in the training sets for the three events, and 2305, 457, and 1677 data texts in the test sets, respectively. After extracting multi-dimensional features from data text, it was necessary to construct an SVM classifier and adjust its parameters to the best state. The trained SVM model was used for predicting the network 
public opinion sentiment tendency of three kinds of events.

\subsection{Hyperparametric analysis}

In this study, ten-fold cross-validation was used to test the setting range of the hyper-parameters of the constructed network public opinion sentiment tendency classification model. The initial hyper-parameter ranges were as follows: the dimension of word vector was 300; the shape of convolution kernel was 2, 3, and 4; the number of corresponding convolution kernels was 100, 100, and 100; dropout was 0.5 ; L2 norm was 0 ; mini_ batch was 32 ; the training time was 200,500, and 200. Using this set of hyperparameters, DG_2018, XS_2019, and BXG_2020. The accuracy rate of emotion orientation recognition was $83.67 \%, 67.83 \%$, and $83.92 \%$, respectively.

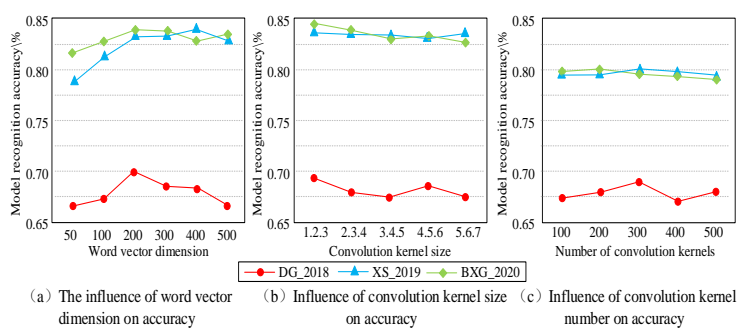

Figure 5: The influence of word vector dimension, convolution kernel size, and number on recognition accuracy.

Following the principle of a single variable, the dimension of the word vector was set as 50, 100, 200, 300, 400 , and 500, respectively, convolution kernel size was 1 , $2,3,2,3,4,3,4,4,5,4,5,6,6$, and the number of convolution kernels was 100, 200, 300, 400, and 500 . Under this set of parameters, the accuracy of identifying the emotional orientation of online public opinion is shown in Figures 5 (a), (b), and (c). When the dimension of word vector changed from 50 to 500, the accuracy of the emotion recognition model first increased and then decreased. When the word vector dimension was 200, the recognition accuracy of the recognition model for three events reached the highest, and the value was higher than that under the initial hyper-parameter setting. When the size of the convolution kernel was $1,2,3$, the recognition model had the highest recognition accuracy for three events. The classification effect of XS_2019 and BXG_2020 events are the best when the number of convolution kernels was 300 . The classification effect of DG_2018 events was the best when the number of convolution kernels was 200. There was no significant difference between the classification effect of XS_ 2019 and BXG_2020 events when the convolution kernel was 200 and 300. The reason for this phenomenon is as follows: when the number of convolution kernels was small, some important features could not be included in the network learning range; when the number of convolution kernels was large, the speed of network training reduced. Under the comprehensive consideration, when the number of convolution kernels was 300 , the recognition effect of the emotion recognition model was the best.

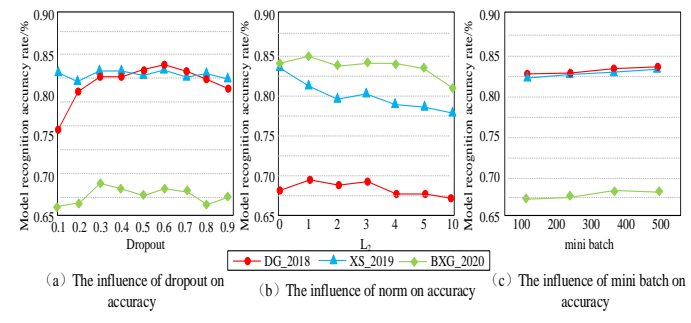

Figure 6: Influence of L2, Dropout value, and mini batch on recognition accuracy.

According to the principle of a single variable, dropout was set as $0.1,0.2,0.3, \ldots, 0.9 ; \mathrm{L} 2$ norm was 0,1 , $2,3,5,10$, and mini_batch was $32,64,96,128$. Under this set of parameters, the accuracy of identifying the emotional tendency of online public opinion is shown in Figures 6 (a), (b), and (c). When dropout was 0.1, 0.2, 0.8, 0.9 , the effect of the emotion recognition model in recognizing BXG-2020 event was poor. The reason for this phenomenon is as follows: when the dropout value was too high, the number of random output neurons was too large, and the probability of fitting was large; when the dropout value was too low, the number of random output neurons was too small to obtain enough neuron characteristics. Therefore, when the dropout value was between 0.3 and 0.7 , the recognition model classification effect was better. The dropout value of 0.6 was selected for the experiment. L2 norm can restrict the model space by constraining the parameters to prevent over-fitting. When the L2 norm was 0 and 1, the classification effect of the recognition model was the best. When the L2 norm value was greater than 3 , the recognition accuracy showed a downward trend. In a certain range, the larger the value of mini_batch was, the higher the utilization rate of model memory was, and the fewer the iterations were. However, under the same accuracy requirements, the time was longer. With the increase of mini_batch value, the corresponding recognition accuracy reaches $100 \%$.

According to the experimental results of the emotion recognition model under different hyper-parameter settings, the optimal hyper parameter settings are as follows: vector dimension was 200 ; dropout was 0.6 ; convolution kernel size was 1, 2, 3; convolution kernel number was 300; L2 norm was 1; mini_batch value was 128. Under this parameter, the recognition accuracy of the emotion recognition model was $85.87 \%, 73.65 \%$, and $85.87 \%$, respectively. Compared with the initial hyperparameter setting, the recognition accuracy was improved by $2.18 \%, 5.82 \%$, and $1.95 \%$, respectively, showing that the classification effect effectively improved.

\subsection{Comparison test results}

In order to verify the feasibility of the network public opinion sentiment orientation recognition model constructed in this study, this study compared the two annotation methods, OCC sentiment annotation and word 
vector representation. The comparison of the recognition effect between the two methods is shown in Figure 7.

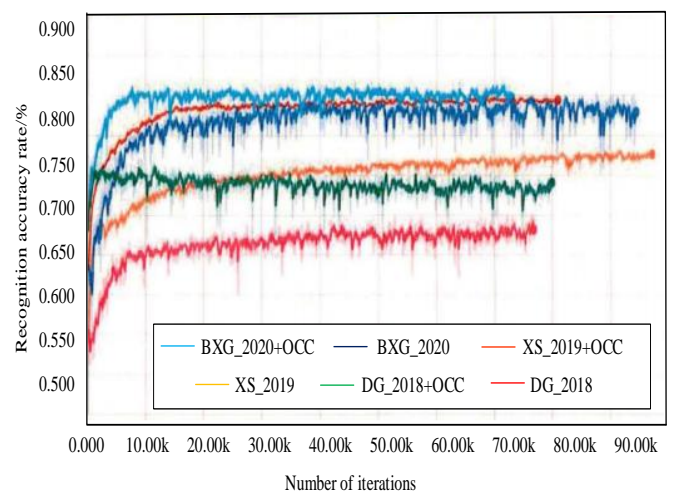

Figure 7: Effect of emotion tagging in OCC on recognition accuracy.

Figure 7 shows the accuracy results of the two annotation methods for identifying three events after 90000 iterations. It was concluded from Figure 7 that when the network public opinion emotion recognition model based on the OCC sentiment annotation method was used for identifying DG_2018, XS_2019, and BXG_2020, the accuracy rates were $84.32 \%, 73.65 \%$, and $85.87 \%$, respectively. The accuracy rates of the artificial tagging method in identifying these three events were $76.38 \%, 65.64 \%$, and $82.35 \%$, respectively. The accuracy of emotion recognition was improved by $3.00 \% \sim 8.00 \%$ by using the OCC emotion tagging method. The above result was because some event reviews in the research database might not express emotion with too many emotive words, the results of manual annotation were lack of standardization, and the emotion classification of some comments by staff was not accurate, which affected the recognition accuracy of subsequent recognition models. The OCC emotion labeling method was based on the concept of machine learning, and complete and correct emotion recognition rules made its annotation accuracy higher than the manual method.

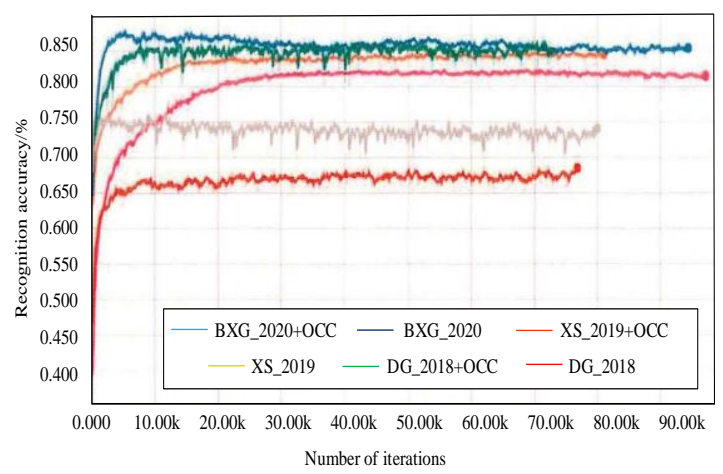

Figure 8: Influence of text representation on recognition accuracy.

In order to compare the effect of word vector generated by Word2Vec on emotion classification, this study used the vocabulary index and the "tf.nn.embedding_lookup" method in TensorFlow to train the word vector from the original data to complete the embedding operation. The two methods were named "cnn_rand" and "cnn_wod2vec". The comparison results are shown in Figure 8 . The accuracy of the model which adopted the cnn_rand method to identify DG_2018, XS_2019, and BXG_2020 events was $84.32 \%, 73.65 \%$, and $85.87 \%$, respectively. The accuracy of the model which adopted the cnn_wod2vec method to identify DG_ 2018, XS_ 2019 and BXG_2020 events was 81.87\%\%, $67.68 \%$, and $86.17 \%$, respectively. The network public opinion emotion recognition model which adopted the cnn_wod2vec method had a better recognition performance in identifying DG_2018 and XS_2019 events; the model which adopted the cnn_rand method was better in identifying BXG_2020 event, and the recognition accuracy of the model was $0.30 \%$ higher than that of the model which adopted the cnn_word2vec method. Overall, the recognition model constructed by generating the word vector with Word2 Vec was better, and the Word2 $\mathrm{Vec}$ method was more suitable for the emotion recognition model constructed in this study.

\begin{tabular}{|l|l|l|l|}
\hline $\begin{array}{l}\text { Classifier/ } \\
\text { event }\end{array}$ & DG_2018 & XS_2019 & BXG_2020 \\
\hline CNN & $84.32 \%$ & $73.65 \%$ & $85.87 \%$ \\
\hline SVM & $66.60 \%$ & $58.20 \%$ & $61.38 \%$ \\
\hline
\end{tabular}

Table 1: Influence of sentiment classifier on the effect of network public opinion sentiment recognition.

The public's emotional tendency to the three events was identified using the CNN classifier and SVM classifier, and the comparison results are shown in Table 1. The data in Table 1 showed that the recognition accuracy of CNN classifier was significantly higher than that of the SVM classifier [24]. It was because the CNN classifier adopted the mode of forward-propagation prediction and back-propagation error, and its unique chain rule improved the output accuracy of the deep neural network model. It was seen from Table 1 that the accuracy rate of the CNN classifier was $17.72 \%, 15.45 \%$, and $24.49 \%$ higher than the SVM classifier. It is inferred that the feature mode of word embedding generated by Word2Vec may not be suitable for text classification using the SVM classifier.

\section{Conclusion}

Unlike traditional machine learning, deep learning has higher accuracy in mining data, and its unique parameter sharing mechanism can significantly reduce the training time of models. In order to improve the efficiency and accuracy of network public opinion sentiment orientation recognition, this study used a convolutional neural network in deep learning to automatically mine network public opinion sentiment. This study was based on the characteristics of a small amount of text and a high frequency of emotional words. The network public opinion sentiment rule base was constructed, and the network public opinion sentiment tendency recognition model was obtained by training with a convolution neural network. The experimental results showed that the OCC emotion tagging method improved the accuracy of emotion recognition by $3.00 \% \sim 8.00 \%$ compared with the 
manual annotation method; compared with the vocabulary index method, Word2Vec was more suitable as the input of the network public opinion emotion recognition model; the accuracy rates of the CNN classifier in identifying DG_2018, XS_2019 and BXG_2020 events were 17.72, $15 . \overline{4}$, and 24.49 percentage points higher than the SVM classifier respectively. The identification model constructed in this study has a high accuracy and can provide information decision support in network public opinion. However, the model has a low accuracy in identifying the emotional orientation of netizens with too little text information data; thus, it is necessary to further optimize the recognition model.

\section{References}

[1] Wu S, Cui X, Hu Y. (2015). Study on the internet public opinion evolution based on SNA. Journal of Sichuan University (Engineering Science Edition), 47, pp. 138-142.

https://doi.org/10.15961/j.jsuese.2015.01.019.

[2] Huang B. (2020). Analyze the Influence of Internet Public Opinion on Public Policy. Open Access Library Journal, 07, pp. 1-9. https://doi.org/10.4236/oalib.1106674.

[3] Song S, Guo Z, Wang X. (2020). The correlation between social transformation economic risk and internet public opinion. Behaviour and Information Technology, pp. 1-11. https://doi.org/10.1080/0144929X.2020.1722750.

[4] Zhang W, Du Y, Li Z, Chen JD. (2018). DeepOpinion: a system for deep analysis and guidance of internet public opinion. Journal of Beijing University of Chemical Technology (Natural Science Edition), 45, pp. 94-98.

[5] Li XZ, Qian YZ, Deng Y, Song WG, Liao JF, Yang MS, Lian YL. (2017). Monitoring and analysis on internet public opinion of agro-products quality and safety in China, 2016. Chinese Science Bulletin, 62, pp. 1095-1102. https://doi.org/10.1360/N97201700015.

[6] Hong W, Li Q, Wu L. (2017). Food safety internet public opinion transmission simulation and management countermeasures considering information authenticity. System Engineering Theory and Practice, 37, pp. 3253-3269. https://doi.org/10.12011/1000-6788(2017)12-325317.

[7] Gesang D, Qiao SJ, Han N, Zhang XS, Yang Y, Yuan CA, Kang J. (2015). An Internet Public Opinion Hotspot Detection Algorithm Based on Single-Pass. Journal of the University of Electronic Science and Technology of China, 44, pp. 599-604. https://doi.org/10.3969/j.issn.10010548.2015.04.021.

[8] Zhang Q. (2019). A Collaborative Group Decision Model for Internet Public Opinion Emergency with Interval Value. American Journal of Modeling and Optimization, 7, pp. 14-19. https://doi.org/10.12691/ajmo-7-1-3.
[9] He H. (2018). Research on prediction of internet public opinion based on grey system theory and fuzzy neural network. Journal of Intelligent and Fuzzy Systems, 35, pp. 1-8. https://doi.org/10.3233/JIFS169591.

[10] Gao X, Fu L. (2017). Methods of uncertain partial differential equation with application to internet public opinion problem. Journal of Intelligent \& Fuzzy Systems, 33, pp. 1-11. https://doi.org/10.3233/JIFS-17409.

[11] Wang A, Liu X, Sun X, Wang J. (2017). Research of internet public opinion based on hybrid algorithm of LDA and VSM. C e Ca, 42, pp. 1508-1513.

[12] Peng ZH. (2016). The Approaches of Internet Public Opinion Research. Libraly Journal, 35, pp. 63-68.

[13] Sang DV, Bao C. (2018). Effective Deep Multisource Multi-task Learning Frameworks for Smile Detection, Emotion Recognition and Gender Classification. Informatica, 42, pp. 345-356. https://doi.org/10.31449/inf.v42i3.2301.

[14] Gjoreski M, Gjoreski H, Kulakov A. (2014). Machine Learning Approach for Emotion Recognition in Speech. Informatica, 38, pp. 377-384.

https://www.researchgate.net/publication/270893946.

[15]Zhang F, Li SC, Guan Y. (2016). Hot issues about drug price in china: from the view of internet public opinion monitoring. Value in Health, 19, pp. A273A274. https://doi.org/10.1016/j.jval.2016.03.1953.

[16]Zhu H, Liu P, Shan X. (2015). Analysis of internetbased public opinion in China, 2012. Journal of Molecular Neuroscience, 49, pp. 614-617. https://doi.org/10.1163/9789004276536_010.

[17] Li L. (2020). Research on the Transfer Rules of Internet Users' Negative Emotional State in Financial Public Opinion. Open Journal of Business and Management, 08, pp. 282-301. https://doi.org/10.4236/ojbm.2020.81017.

[18] Li L, Zhu X, Hao Y, Wang S, Gao X, Huang Q. (2019). A Hierarchical CNN-RNN Approach for Visual Emotion Classification. ACM Transactions on Multimedia Computing Communications and Applications, 15, pp. 1-17. https://doi.org/10.1145/3359753.

[19] Ni HB. (2020). Face Recognition Based on Deep Learning Under the Background of Big Data. Informatica 44, pp. 491-495.

[20] Gupta A, Srinivasan SM. (2020). Constructing a Heterogeneous Training Dataset for Emotion Classification. Procedia Computer Science, 168, pp. 73-79. https://doi.org/10.1016/j.procs.2020.02.259.

[21]Zhang W, He X, Lu W. (2020). Exploring Discriminative Representations for Image Emotion Recognition With CNNs. IEEE Transactions on Multimedia, 22, pp. 515-523. https://doi.org/10.1109/TMM.2019.2928998.

[22] Rao Y, Xie H, Li J, Jin F, Wang F, Li Q. (2016). Social emotion classification of short text via topiclevel maximum entropy model. Information \& Management, 53, pp. 978-986. https://doi.org/10.1016/j.im.2016.04.005. 
[23]Lin YP. (2020). Constructing a Personalized CrossDay EEG-Based Emotion-Classification Model Using Transfer Learning. IEEE Journal of Biomedical and Health Informatics, 24, pp. 12551264. https://doi.org/10.1109/JBHI.2019.2934172.

[24] Wang D, Xu G. (2020). Research on the Detection of Network Intrusion Prevention With Svm Based Optimization Algorithm. Informatica, 44, 269-273. https://doi.org/10.31449/inf.v44i2.3195. 